\title{
Hans Kelsen und die Berufungen nach Graz, Czernowitz und Wien 1916-1919*
}

Hans Kelsen, one of the leading jurists of the 20th century, habilitated himself in 1911 and became associate professor at University of Vienna in July 1918. Very soon after that, in July 1919, he became full professor. So it seems that the first steps of his career were very difficult for him and only the political circumstances - the collapse of the monarchy and the rising of the republic in 1918 - enabled the further steps. This article, however, shows that Kelsen was in discussion for a professorship even earlier, in 1916 at the University of Graz and soon after that in Czernowitz, before he was appointed at the University of Vienna. It gives a panorama view of the situation of the public law in Austria at the beginning of the $20^{\text {th }}$ century.

Im Gegensatz zu vielen anderen Rechtswissenschaftern seiner Zeit musste Kelsen wenigstens zu Beginn seiner akademischen Karriere kein „akademisches Nomadentum" erleiden; vielmehr ist er das typische Beispiel für eine sog. Hausberufung: Geboren in Prag, aber aufgewachsen in Wien, ging er hier zur Schule und studierte die Rechts- und Staatswissenschaften, habilitierte sich 1911 an der Alma Mater Rudolphina und wurde zunächst, 1918, außerordentlicher, dann, 1919, ordentlicher Professor an der Universität Wien. Erst 1930, kurz vor seinem 49. Geburtstag, verließ er Wien, um einen Ruf an die Universität Köln anzunehmen. Damit allerdings begann für ihn eine zehnjährige Odyssee, die ihn über Genf und Prag

\footnotetext{
* Die Arbeit enthält Ergebnisse gleich zweier von mir geleiteter Forschungsprojekte: dem FWF-Projekt P 19287 „Biographische Untersuchungen zu Hans Kelsen in den Jahren 1881-1940" sowie dem FWFProjekt P 21280 „Die Wiener Rechts- und Staatswissenschaftliche Fakultät 1918-1938“. Für wertvolle Hilfe bei der Beschaffung der zitierten Archivalien danke ich Herrn Mag. Jürgen BusCH LL.M. sowie dem Leiter des Grazer Universitätsarchives, Herrn Univ.Prof. Dr. Alois KeRNBAUER.
}

1940 in die USA führte, wo er zunächst in Harvard lehrte, um endlich in Berkeley in Kalifornien eine dauerhafte Bleibe zu finden.

Schon vor 1930 zeigten viele Universitäten immer wieder Interesse, den Begründer der Reinen Rechtslehre in ihre Mitte zu holen, ${ }^{1}$ und dies beileibe nicht erst, als Kelsen bereits allgemein zu den bedeutendsten Juristen weltweit gezählt wurde. Namentlich an der Karl-FerdinandsUniversität zu Graz und der Franz-Josephs-Universität zu Czernowitz gab es schon während des Ersten Weltkrieges Ambitionen, Kelsen zu berufen. Warum es dann letztlich nicht dazu kam und wie sich die Berufung an die Universität Wien letztlich gestaltete, soll im Mittelpunkt dieses Beitrages stehen.

\section{Der junge Privatdozent}

Kelsens Habilitierung war reichlich unspektakulär verlaufen. In seiner Autobiographie

\footnotetext{
${ }^{1}$ Nur beispielhaft seien die Bemühungen der Universität Frankfurt 1925 genannt; vgl. dazu HAMMERSTEIN, Frankfurt 128f.; SCHMITZ, Karl Renners Briefe 144.
} 
schreibt er, dass "sich mit einem ausfuehrlichen Gutachten nur der Professor fuer Rechtsgeschichte Baron Schwind" dagegen ausgesprochen hatte, Hans Kelsen die venia legendi für „allgemeines und österreichisches Staatsrecht, Rechtsphilosophie und deren Geschichte" zu verleihen, doch selbst dies ist nicht korrekt, sondern beruht auf einer Verwechslung mit Kelsens späterer Ernennung zum ao. Professor, auf die noch zurückzukommen ist. ${ }^{2}$ In Wahrheit dürfte es so gewesen sein, dass weder der Erstgutachter, Prof. Adolf Menzel, noch der Zweitgutachter, Prof. Edmund Bernatzik, die ganze Tragweite von Kelsens bahnbrechender Habilitationsschrift, „Hauptprobleme der Staatsrechtslehre" erkannt hatten; Kelsen selbst mutmaßte sogar, dass Bernatzik die Schrift niemals gelesen hatte, sondern, wie damals üblich, sich lediglich dem positiven Befund des Erstgutachters Menzel angeschlossen hatte. ${ }^{3}$ Bis zu einem gewissen Grad war Kelsen daran selbst schuld: Seine Buch überstieg mit über 700 Seiten andere Habilitationsschriften seiner Zeit um ein Vielfaches, die vollständige Lektüre desselben war geradezu eine Zumutung, zumal die trockenen rechtstheoretischen Ausführungen an keiner Stelle übersichtlich zusammengefasst oder sonst plakativ dargestellt wurden. Vielmehr entwickelte Kelsen seine eigenen, durchaus revolutionären Ansichten immer erst nach seitenlangen Auseinandersetzungen mit nahezu der gesamten Staatsrechtslehre seiner Zeit. ${ }^{4}$

\footnotetext{
${ }^{2}$ KELSEN, Autobiographie 43; vgl. dazu schon OLECHOWSKI, Rechtsphilosophie 431f.

${ }^{3}$ KELSEN, Autobiographie 43. Der Habilitationsakt ist in ÖStA, AVA, Unterricht Allg., Univ. Wien, Kart. 611, Personalakt Kelsen Hans, erhalten, nicht jedoch die beiden Gutachten. Siehe zur Situation der deutschsprachigen Staatsrechtslehre $\mathrm{zu}$ jener Zeit STOLLEIS, Geschichte II, zur Situation in Österreich bes 306 ff; für die Universität Wien vgl. WALTER, Lehre Wien $615 \mathrm{ff}$ und nunmehr auch OLECHOWSKI, EHS, STAUDIGL-CiECHOWICZ, Fakultät 476.

${ }^{4}$ JESTAEDT, Editorischer Bericht $902 \mathrm{ff}$.
}

Daher brauchte es einige Jahre, bis das gesamte revolutionäre Potential von Kelsens Lehre erkannt wurde; die verschiedenen Rezensionen, die in der Folge zu seinem Buch erschienen, mögen das ihre dazu beigetragen haben, mehr aber wohl noch seine eigenen Vorträge und kleineren Schriften, die in den folgenden Jahren erschienen, so etwa sein Vortrag „Über Grenzen zwischen juristischer und soziologischer Methode",5 den er noch kurz vor seiner Habilitierung in der Soziologischen Gesellschaft in Wien gehalten hatte, und der noch im selben Jahr 1911 als Broschüre erschien, besonders aber die Kontroverse mit Eugen Ehrlich.

Der berühmte Czernowitzer Professor hatte 1913 sein Buch "Grundlegung der Soziologie des Rechts" veröffentlicht, in der er der von ihm mitbegründeten Disziplin Rechtssoziologie eine überragende Bedeutung für die gesamten Rechtswissenschaften gegeben hatte. Seiner Ansicht nach nahm das Recht seinen Ausgang aus gewissen tatsächlichen Verhältnissen, den „Rechtstatsachen“: So sei etwa die Familie „älter als die Familienordnung, [...] es gab Verträge, bevor noch ein Vertragsrecht vorhanden war, und selbst das Testament, wo es urwüchsig entstanden ist, reicht über das Testamentsrecht hinaus." ${ }^{\circ}$ Aufgabe der Juristen müsse es daher sein, jene tatsächlich befolgten „Regeln des Handelns" zu untersuchen, aus denen das Recht fließe, wobei es sich dabei ,selbstverständlich [um] eine Regel, nach der nicht nur in der Regel gehandelt wird, sondern auch gehandelt werden soll“ drehe. ${ }^{7}$ Hier nun hakte Kelsen ein und erklärte diese Gleichsetzung für „offenbar falsch! Denn jene Regel, die von einer kausal-erklärenden Betrachtugung aufgesucht wird, ist nichts als eine Seinsregel, die darüber, was soll, gar keine Auskunft gibt." 8

\footnotetext{
${ }^{5}$ KELSEN, Über Grenzen.

${ }^{6}$ EHRLICH, Grundlegung 28.

${ }^{7}$ EHRLICH, Grundlegung 7.

${ }^{8}$ KeLSEN, Grundlegung 323.
} 
Bekanntlich führte die Kelsen-Ehrlich-Kontroverse dazu, dass Ehrlich auf Kelsens Rezension zunächst eine "Entgegnung" schrieb und als Kelsen seinerseits eine "Replik“ veröffentlichte, auf diese selbst mit einer "Replik" antwortete, auf welche Kelsen partout ein "Schlusswort" setzen musste. ${ }^{9}$

Die Verbissenheit, mit der beide Wissenschafter ihre Kontroverse führten, wie auch der Tonfall ihrer Schriften (so behauptete Ehrlich, Kelsen habe versucht, ihn „,nahezu für einen Idioten zu erklären“10, während Kelsen schreibt, es „nimmt mich nicht Wunder" wenn „Ehrlich meinen Einwand nicht versteht"11) sind, auch gemessen an anderen Kontroversen jener Zeit, bemerkenswert. Der literarische Streit Kelsen-Ehrlich ist umso merkwürdiger, wenn man sich vor Augen hält, dass auf der einen Seite ein 54-jähriger, angesehener Professor und vormaliger Universitätsrektor, auf der anderen Seite ein gerade 35jähriger, völlig unbekannter Privatdozent steht. Dies kann nicht einfach damit begründet werden, dass Ehrlichs wissenschaftliches Konzept geradezu das Gegenteil von Kelsens Grundprinzip - der Trennung von Sein und Sollen - war; dass Eugen Ehrlich also gerade jene "Grenzen zwischen juristischer und soziologischer Methode" überschritten hatte, die Hans Kelsen zuvor so penibel gezogen hatte. Hinter seinem Engagement steckte möglicherweise auch das taktische Kalkül, dass er durch eine derartige Kritik entsprechende Aufmerksamkeit in der scientific community ernten würde. Wenn dem so war, dann ging Kelsens Rechnung tatsächlich auf. Denn wie gleich zu zeigen sein wird, erwarb sich Kelsen innerhalb kürzester Zeit einen Ruf, der groß genug war, dass er als aussichtsreicher Kandidat für einen staatsrechtlichen Lehrstuhl gehandelt wurde.

\footnotetext{
${ }^{9}$ EHRLICH, Entgegnung; KELSEN, Replik; EHRLICH, Replik; KELSEN, Schlusswort.

${ }^{10}$ EHRLICH, Entgegnung 57.

${ }^{11}$ Kelsen, Replik 611.
}

Dazu mochte auch beitragen, dass Kelsen bereits unmittelbar nach seiner Habilitation daran ging, eine eigene "Schule" auszubilden, indem er einen Kreis von fortgeschrittenen Studenten und Absolventen um sich sammelte, mit denen er Privatseminare veranstaltete; schon bald, nämlich 1915, ging aus diesen Treffen ein weiterer Privatdozent hervor, nämlich Leonid Pitamic. ${ }^{12}$ Nach dem Krieg sollte es Kelsen gelingen, praktisch im Jahresrhythmus einen weiteren seiner Schüler - Adolf J. Merkl, Fritz Sander, Alfred Verdroß und andere - zu habilitieren. Zuletzt ist auch daran zu erinnern, dass Kelsen auch in der Volksbildung tätig war, sodass er sich auch außerhalb der Universität schon bald einen Namen machte. ${ }^{13}$

Insoweit scheint es kaum glaubhaft, wenn Kelsen in seiner Autobiographie schreibt, dass er noch 1915 keinerlei Ahnung davon hatte, dass er schon bald die Karriereleiter emporklimmen werde. In jenem Jahr hatte er - „der ueblichen Routine gemaess - den Titel eines a.o. Professors" erhalten; und diese Position musste ich eigentlich bei meiner juedischen Abstammung als das aeusserste ansehen das mir unter den gegebenen Verhaeltnissen erreichbar war."14 Tatsache ist allerdings, dass die Fächer Staatsund Verwaltungsrecht - gemessen an ihrer damals noch bescheidenen Verankerung im Studienplan - sehr stark an der Fakultät vertreten waren, nämlich mit zwei Ordinarien (Edmund Bernatzik und Adolf Menzel), einem Extraordinarius (Rudolf von Laun) und nicht weniger als sieben Privatdozenten, die sich noch vor Kelsen habilitiert hatten und sich teils in sehr angesehener Position befanden;15 die Titelverleihung von 1915 kann tatsächlich als ein Routineakt angesehen werden, als mit allerhöchster

\footnotetext{
12 Vgl. dazu PAVČNIK, Pitamic sowie den Beitrag desselben Autors in diesem Band.

${ }^{13}$ Ausführlich EHS, Kelsen.

${ }^{14}$ KELSEN, Autobiographie 54.

${ }^{15}$ WALTER, Lehre Wien $619 \mathrm{ff}$.
} 
Entschließung vom 21. September dieses Jahres gleich fünf Staatsrechtler zugleich geehrt wurden, ohne dass mit diesen Ehrungen irgendwelche dienstrechtliche Konsequenzen verbunden waren: Der Hofrat des Verwaltungsgerichtshofes Priv.Doz. Dr. Rudolf Hermann von Herrnritt und der Professor an der Technischen Hochschule Wien Dr. Josef Redlich erhielten die Titel von ordentlichen Universitätsprofessoren, während der Titel eines außerordentlichen Universitätsprofessors außer an Kelsen auch noch an den Ministerialsekretär im Handelsministerium Priv.Doz. Dr. Fritz Hawelka und an den Ministerialvizesekretär im selben Ministerium Priv.Doz. Dr. Leo Wittmayer vergeben wurden. ${ }^{16}$

\section{Graz}

Etwa zwei Monate später, am 11. November 1915, starb in Baden bei Wien der Ordinarius des Staatsrechtes an der Karl-Franzens-Universität Graz Franz Hauke. Haukes Lebenslauf ist typisch für die langen akademischen Karrierewege in jener Zeit: Geboren in Mauer bei Wien, hatte er sich 1884 in Innsbruck habilitiert und war ein Jahr später an die Universität Czernowitz berufen worden, der er im akademischen Jahr 1900/01 als Rektor vorstand. 1904 erfolgte die Berufung an die Universität Graz, wo er ebenfalls - im Jahr 1911/12 - das Rektorat bekleidete. Wissenschaftlich hatte er sich v.a. mit dem Verhältnis zwischen Monarchen und Parlament beschäftigt, auch ein „Grundriß des Verfassungsrechts" stammte aus seiner Feder. ${ }^{17}$ Haukes Tod im 63. Lebensjahr riss eine Lücke in

\footnotetext{
${ }^{16}$ A.h. Entschließung vom 21. 9. 1915 Z 28493, in ÖStA, AVA Unterricht allg., Univ. Wien, Kart. 610, Personalakt Herrnritt; vgl. auch Wiener Zeitung Nr. 219 vom 22. 9.1915, 1 (amtlicher Teil). Biographische Angaben zu den Genannten bei OLECHOWSKI, EHS, STAUDIGL-CiECHOWICZ, Fakultät 473.

${ }^{17}$ ANONYMUS, Hauke; WALTER, Lehre Graz 552f.
}

die Reihen der Fakultät, die so rasch als möglich geschlossen werden musste. Bereits zwei Wochen nach seinem Hinscheiden wurde das Professorenkollegium in Graz vom Unterrichtsministerium eingeladen, Vorschläge zur Nachbesetzung zu erstatten. Eine fünfköpfige Berufungskommission wurde gebildet und innerhalb derselben das Kommissionsmitglied Max Layer, neben Hauke der zweite Ordinarius für Staatsrecht in Graz, beauftragt, die Universitätslandschaft auszuloten und der Kommission zu berichten. ${ }^{18}$

Layer legte seinen schriftlichen Bericht am 23. Februar 1916 vor und nannte nacheinander drei ordentliche und drei außerordentliche Professoren sowie vier Privatdozenten, die für die Stelle in Frage kämen, wobei er jedoch sofort betonte, dass es nicht "auf die derzeitigen Rangverhältnisse“, sondern einzig auf die "Tüchtigkeit" der einzelnen Persönlichkeiten ankäme. ${ }^{19}$ Die Liste bestand ausschließlich aus Österreichern; eine Berufung aus Deutschland lehnte Layer sowohl mit Rücksicht auf das zu betreuende Fach als auch aus finanziellen Gründen ab. ${ }^{20}$ Jede Person wurde einzeln skizziert und die wichtigsten Schriften genannt sowie abschließend gewürdigt.

Bei den drei Ordinarien handelte es sich um Ludwig Spiegel aus Prag, Max Kulisch aus Innsbruck und Otto Dungern aus Czernowitz. Layer fügte allerdings gleich hinzu, dass der bereits 52 Jahre alte Spiegel ${ }^{21}$ zwar höchst qualifiziert sei,

\footnotetext{
${ }^{18}$ Bericht des Dekans Josef von Anders an das Ministerium vom 8. 3. 1916, in: UA Graz, Jur. Fak. Z. 304.

${ }^{19}$ Bericht betreffend die Besetzung der Lehrkanzel für Staats- und Verwaltungs-Recht vom 23. 2. 1916, in: UA Graz, Jur. Fak. Z. 304.

${ }^{20}$ Nichtsdestoweniger würdigte Layer auch den gebürtigen Österreicher Julius Hatschek, der damals als ao. Professor in Göttingen wirkte, kam aber auch hier zum Schluss, dass sich dieser „seit Beginn seiner akademischen Laufbahn" nicht mehr mit österreichischem Recht beschäftigt habe; vgl. ebd.

${ }^{21} \mathrm{Vgl}$. zu ihm zuletzt OBERKOFLER, Spiegel.
} 
jedoch kaum aus Prag weggehen würde, weshalb er nicht in den ",engeren Vorschlag“ kommen solle. Ausführlich würdigte er dagegen Kulisch: Dieser, 1870 in Bodenbach in Böhmen [Děčín-Podmokly] geboren, hatte in Wien die Rechtswissenschaften studiert und anschließend auch Jellinek in Heidelberg und Preuß in Berlin besucht, bevor er 1900 in den politischen Verwaltungsdienst (zunächst Böhmens, dann Tirols) trat und sich parallel dazu an der Universität Prag habilitierte. 1906 wurde Kulisch zum außerordentlichen, 1909 zum ordentlichen Professor an der Universität Innsbruck ernannt. ${ }^{22} \mathrm{Er}$ besaß damit, wie Layer lobend hervorhob, eine „Kenntnis der Praxis", welche ihn „vor Einseitigkeiten der juristischen Methode" bewahre und verliere "auch bei theoretischen Konstruktionen niemals den realen Boden des positiven Rechts, das er gründlich beherrscht. “23

Geradezu begeistert zeigte sich Layer vom gebürtigen Preußen Otto von Dungern, der sich 1909 in Graz habilitiert hatte und seit 1911 als ao. Professor in Czernowitz wirkte. Dungern war primär rechtshistorisch tätig, und weil zu jener Zeit auch eine Professur für Rechtsgeschichte an der Universität Graz vakant war, meinte Layer, dass Dungern dort "ganz besonders an seinem Platze wäre", aber auch in Bezug auf den staatsrechtlichen Lehrstuhl „unmöglich übergehen" werden könne; es handle sich eben um einen in beiden Materien bewanderten Wissenschafter, „der zwar ungeachtet der Vielseitigkeit seines Schaffens von einer gewissen Einseitigkeit in der Behandlung seiner Probleme nicht ganz freizusprechen ist, aber stets interessant und wirklich schöpferisch wirkt. “24

Kritisch äußerte sich Layer dagegen zu Rudolf von Laun, der in Wien eine außerordentliche Professur bekleidete: Sein wissenschaftliches Hauptwerk, „Das freie Ermessen und seine

\footnotetext{
${ }^{22}$ HeKajllo, Kulisch 341.

${ }^{23}$ Bericht (wie Anm. 19) 3.

${ }^{24}$ Bericht (wie Anm. 19) 5.
}

Grenzen“ sei in der Literatur „sehr scharf, vielleicht etwas zu scharf, aber nicht ungerecht kritisiert worden". Dass diese Kritik vor allem von Kelsen gekommen war, verschwieg Layer, schloss sich ihr aber inhaltlich an, indem er meinte, dass Launs Arbeit „in der Hauptsache verfehlt" sei, "weil sich der Autor zu einer einheitlichen Auffassung nicht durchringen konnte“. ${ }^{\prime \prime}$

Von den Privatdozenten hob Layer besonders Hans Kelsen hervor: Bei seiner Habilitationsschrift handle es sich um ein "fundamentales, tief durchgedachtes Werk", auch wenn die „Endergebnisse vergleichsweise dürftig sind [...] es wird ein logisch entwickelter, künstlich konstruierter Rechtsstaatsbegriff aufgebaut, wie er im Grunde nirgends, wohl auch in Oesterreich nicht existiert. Das vermag aber nichts an dem Urteil zu ändern, dass wir ein wahrhaft grosszügiges Werk vor uns haben, an dem kein Staatsrechtslehrer, überhaupt kein Jurist achtlos vorübergehen kann.“" Layer lobte auch die übrigen Arbeiten Kelsens und hob hervor, dass dieser ",in Wien ausserordentliche Lehrerfolge aufzuweisen“ und namentlich „einen bei einem Privat-Dozenten noch kaum dagewesenen $\mathrm{Zu}$ lauf von Hörern" vorweisen könne, was auf eine "hervorragende Lehrbefähigung" schließen lasse. ${ }^{26}$

Zusammenfassend urteilte Layer: „für die genialste Begabung halte ich Frhr. v. Dungern, für den reifsten akademischen Lehrer, der auch für

\footnotetext{
${ }^{25}$ Bericht (wie Anm. 19) 8. Die Monographie Launs zum freien Ermessen war 1910 erschienen und hatte Kelsen veranlasst, in seine bereits im Satz befindliche Habilitationsschrift einen umfangreichen Exkurs aufzunehmen, in dem er sich mit Laun auseinandersetzte, vgl. KELSEN, Hauptprobleme 651-660, und JESTAEDT, Editorischer Bericht 893. Laun unterschied insbesondere zwischen "objektiven, inneren" und „äußeren, gesetzlichen“ Schranken des freien Ermessens, während Kelsen nachwies, dass zwischen diesen beiden Arten kein Unterschied bestand.

${ }^{26}$ Bericht (wie Anm. 19) 8f.
} 
den ganzen Umfang des Faches die allseitige Eignung mitbringt, Kulisch, für den tiefsten Denker Kelsen. “ 27

Layer schlug daher vor, alle drei gleichrangig aequo loco - in den Berufungsvorschlag aufzunehmen. Die Berufungskommission entschloss sich jedoch, Kulisch primo loco und die beiden anderen secundo loco zu reihen. In dieser Form gelangte der Antrag ins Fakultätskollegium, wo am 2. März 1916 eine heftige Debatte stattfand, von der wir nur wissen, dass Kelsen der Hauptstreitgegenstand war. ${ }^{28}$ Sowohl, ob er überhaupt genannt werden solle, als auch seine Reihung waren überaus umstritten, die Argumente für und wider sind nicht bekannt. Schließlich wurde Kulisch an erster, Dungern an zweiter und Kelsen an dritter Stelle gereiht. ${ }^{29}$

Max Layer verfasste hierauf ein Minoritätsvotum, welches v.a. deshalb auffällig ist, weil er darin betonte, dass seine Beurteilung der genannten Personen alleine aufgrund „einer genauen Kenntnis ihrer Werke als der einzig zulässigen objektiven Grundlage" beruhe und er allein bestrebt war, „die Tüchtigsten an erster Stelle zu nennen." ${ }^{30}$ War ihm vorgeworfen worden, aus persönlichen Motiven gehandelt zu haben? Es ist dies umso erstaunlicher, als persönliche Kontakte zwischen Layer und Kelsen aus der Zeit vor 1916 nicht bekannt sind. ${ }^{31}$

Das Minoritätsvotum wurde vom Rechtshistoriker Paul Puntschart und vom Ökonomen

\footnotetext{
${ }^{27}$ Bericht (wie Anm. 19) 13f. Gewürdigt wurden im Gutachten Layers auch noch Hans v. Frisch (Czernowitz), Julius Hatschek (Göttingen), Hans Nawiasky (Wien), Fritz Hawelka (Wien) und Leo Wittmayer (Wien).

${ }^{28}$ Bericht des Dekans (wie Anm. 18) 6.

${ }^{29}$ GOLLER, Rechtsphilosophie 186.

${ }^{30}$ Das Separatvotum in UA Graz, Jur. Fak. Z. 520.

${ }^{31}$ Ausgenommen der Umstand, dass Layer als damaliger Professor der Universität Wien am 7.5. 1906 dem Prüfungssenat von Kelsens „Romanum“ angehört hatte: Universitätsarchiv Wien, Jur. Rig. Protocoll J 13.16 1904-1905, Zahl 1593.
}

Josef Schumpeter unterstützt, blieb jedoch wirkungslos. Der Ruf ging an Otto von Dungern, der mit Oktober 1916 sein neues Amt antrat. Auch er ist, so wie sein Vorgänger Hauke, ein Beispiel für die langen Karrierewege jener Zeit: Geboren 1875 in Neuwied in der preußischen Rheinprovinz, hatte er in Erlangen promoviert und sich, wie erwähnt, in Graz habilitiert, worauf er nach Czernowitz gegangen war, um fünf Jahre später von dort zurückzukommen. In Graz lehrte er bis zu seiner Emeritierung im Jahre 1941.32

\section{Czernowitz}

Damit wurde in Czernowitz ein Extraordinariat frei - was zu jener Zeit allerdings nicht viel heiBen mochte: Denn der Vorlesungsbetrieb an Österreichs östlichster Universität war seit Kriegsbeginn eingestellt, das Rektorat nach Wien verlegt worden, die Professoren waren beurlaubt und lebten an den verschiedensten Orten in den westlichen Provinzen der Monarchie. Wie unter diesen Umständen noch Fakultätsbeschlüsse zustande kamen - etwa im Umlaufwege? - ist unbekannt; aber Tatsache ist, dass der Minister zwei Jahre später dem Kaiser berichtete, das Professorenkollegium der Universität Czernowitz hätte "einstimmig“ einen Besetzungsvorschlag für die Nachfolge nach Otto von Dungern eingebracht, mit Hans Kelsen an erster und Leonidas Pitamic an zweiter Stelle. ${ }^{33}$ Weitere Hintergründe kennen wir leider nicht.

Wie das Grazer Beispiel zeigt, basierten solche Besetzungsvorschläge in aller Regel auf Referaten der dem Fach am nächsten stehenden Professoren; im Fall von Czernowitz war dies der

\footnotetext{
32 WALTER, Lehre Graz 553.

${ }^{33}$ Vortrag vom 25. 6. 1918, ÖStA, AVA, Unterricht Allg., Univ.Wien, Karton 612, Personalakt Pisko Oskar, Z 23478/1918.
} 
einzige Ordinarius für Staatsrecht Hans Ritter von Frisch, der also gleich zwei Vertreter der Reinen Rechtslehre in seinen Vorschlag aufgenommen hatte, obwohl er sich selbst in seinen Schriften nicht als Anhänger dieser Theorie erwies. ${ }^{34}$ "Einstimmig" müsste auch bedeuten, dass der Beschluss vom Romanisten Eugen Ehrlich mitgetragen worden war, was ebenfalls sonderbar anmutet. Die schlechte Quellenlage erlaubt uns hier leider keine weiteren MutmaBungen.

\section{Wien}

In der kaiserlichen Entschließung, mit der Hans Kelsen 1915 zum Titularprofessor ernannt worden war, war er als "Adjunkt" an der Exportakademie bezeichnet; tatsächlich übte er diese Tätigkeit seit Kriegsbeginn nicht mehr aus, sondern wirkte zu jener Zeit als Stellvertreter des Militäranwaltes des k.u.k. Militärkommandanten von Wien. ${ }^{35}$ Wenige Tage später, am 1. Oktober, wurde er in die Justizabteilung des k.u.k. Kriegsministeriums versetzt, wo er eine bemerkenswerte Karriere absolvierte. ${ }^{36} \mathrm{Ob}$ es Glück war oder auch Geschick - jedenfalls wurde Kelsen im Oktober 1917 dem Präsidialbüro des Ministers, Rudolf Stöger-Steiner, zugewiesen, und dieser war mit seinen Leistungen äußerst zufrieden. Kelsen berichtet, dass ihm Stöger-Steiner sogar anbot, auch nach Kriegsende in einer leitenden Position im Ministerium $\mathrm{zu}$ verbleiben; und auch das Sozialministerium hatte ihm „ein sehr guenstiges Angebot" ge-

\footnotetext{
${ }^{34} \mathrm{Vgl}$. zu ihm zuletzt OlechOWSKI, EHS, STAUDIGLCiechowicz, Fakultät 683.

${ }^{35}$ BusCH, Kelsen 64.

${ }^{36}$ Dieser Karriereabschnitt ist von OBERKOFLeR, RABOFSKY, Kelsen, sehr ausführlich, jedoch ganz offensichtlich cum ira et studio verfasst worden. Ein anderes, differenzierteres und wohl treffenderes Bild von den Umständen, die Kelsen ins das Ministerkabinett zeichnet BusCH, Kelsen im Weltkrieg, besonders 68.
}

macht. In dieser Situation aber ging Kelsen zu Bernatzik, um seine "Chancen an der Universitaet fest[zu]stellen. Ich [...] sagte ihm, dass ich keines dieser Angebote anzunehmen beabsichtigte, wenn ich eine wirkliche ausserordentliche Professur erhalten wuerde. [...] Wie gross war mein Erstaunen, als Bernatzik - als Antwort mich einfach fragte, fuer welche Faecher ich die Professur haben wolle. [...] Ich zweifle nicht daran dass Bernatzik's Haltung ganz entschieden durch meine Stellung im Kriegsministerium bestimmt war. Diese Stellung hatte auf ihn mehr Eindruck gemacht als alle meine bisherigen Publikationen.“ ${ }^{37}$ Tatsächlich beantragten in weiterer Folge sowohl die Fakultät als auch das k.u.k. Kriegsministerium beim k.k. Unterrichtsministerium eine außerordentliche Professur für Staatsrecht mit besonderer Berücksichtigung des Militärrechts. ${ }^{38}$

Am 27. Februar 1918 wurde ein Komitee, bestehend aus dem Dekan Hans von Voltelini, den Staatsrechtlern Bernatzik und Menzel, dem Zivilprozessualisten Hans Sperl sowie dem Rechtshistoriker Ernst von Schwind gebildet. Nun aber formierte sich ernsthafter Widerstand gegen einen weiteren Karrieresprung Kelsens: Schwind, der sich bis dahin niemals mit Fragen der Rechtstheorie auseinandergesetzt hatte, sprach sich gegen die Ernennung Kelsens aus, und als er von den übrigen Mitgliedern des Komitees überstimmt wurde, verfasste er ein Minoritätsvotum, in dem er Kelsens Lehre als "destruktiv und zersetzend, im Rahmen der akademischen Lehre für die Studierenden vielfach blendend, aber im Erfolge verwirrend und höchst bedenklich“ bezeichnete. ${ }^{39}$

\footnotetext{
${ }^{37}$ KeLSEN, Autobiographie 54.

${ }^{38}$ Schreiben des k.u.k. Kriegsministers Stöger-Steiner an den k.k. Unterrichtsminister vom 7. 3. 1918, ÖStA, AVA. Unterricht Allg., Univ. Wien, Kart. 607.

${ }^{39}$ ÖStA. AVA, Unterricht Allg., Univ. Wien, Kart. 610, Personalakt Herrnritt Rudolf, Z 9583/1918. Vgl. dazu schon OleCHOWSKI, Rechtsphilosophie gegen Rechtsgeschichte 432 .
} 
Und auch von anderer Seite drohte Gefahr: In der Militärkanzlei des Kaisers wirkte damals Major Albin Schager, der sich in Innsbruck für Militärstrafprozessrecht habilitiert hatte und nun ebenfalls Ambitionen auf die neue Professur in Wien hatte. „Kelsen ahnte nichts von all dem, sondern erfuhr es durch einen Zufall, als er auf der Rückfahrt vom kaiserlichen Hauptquartier in Baden nach Wien in der ,Elektrischen' den damaligen Unterrichtsminister [Ludwig] Ćwikliński traf. Dieser sagte ihm, daß seine Ernennung, die er selbst befürwortet hatte, doch nicht erfolgen könne, weil sich eine sehr hohe Persönlichkeit [der Chef der kaiserlichen Militärkanzlei, General Ferdinand von Marterer] für einen anderen Kandidaten einsetzte. “40 Kelsen wandte sich sofort an den Kriegsminister, der ein Schreiben an das Unterrichtsministerium richtete und damit den Weg für Kelsen ebnete. ${ }^{41}$ "Rückendeckung" bekam er dabei auch vom k.k. Landesverteidigungsminister Karl Czapp von Birkstetten, der klarmachte, dass das neue Extraordinariat an der Universität Wien ja nicht das ganze Militärrecht, sondern lediglich das Militärverfassungs- und Militärverwaltungsrecht (also den Bereich, in dem Kelsen Erfahrungen gesammelt hatte) umfassen solle, zumal ja schon die Professoren Alexander Löffler und Georg Lelewer Vorlesungen aus Militärstrafrecht hielten (und somit der Militärstrafrechtler Schager entbehrlich sei). ${ }^{42}$

\footnotetext{
${ }^{40}$ MÉTALL, Kelsen 28. Tatsächlich hatte der Chef der Militärkanzlei am 22. 3. 1918 ein Schreiben an den k.k. Ministerpräsidenten gerichtet, in dem er sich für Schager einsetzte: Abschrift in ÖStA, AVA, Unterricht Allg., Univ. Wien, Kart. 607.

${ }^{41}$ Schreiben des k.u.k. Kriegsministers Rudolf StögerSteiner vom 25. 4. 1918, ÖStA, AVA, Unterricht Allg., Univ. Wien, Kart. 607; vgl. auch KelseN, Autobiographie 55.

${ }^{42}$ Schreiben des k.k. Landesverteidigungsministers Karl Czapp vom 22. 3. 1918, ÖStA, AVA, Unterricht Allg., Univ. Wien, Kart. 607.
}

Die neu geschaffene Professur war somit eine hochpolitische Angelegenheit, an der - mit Hinzuziehung des k.k. Finanzministeriums, das die neue Planstelle ja auch genehmigen musste nicht weniger als vier Ministerien sowie die k.u.k. Militärkanzlei beteiligt waren!

In seinem Vortrag, den Ćwikliński zwei Monate später, am 26. Juni 1918, vor dem Kaiser hielt, war von all den Intrigen nichts mehr zu bemerken. Er berichtete nur ganz allgemein, dass sowohl die "Kriegsverwaltung" als auch die Wiener Juristenfakultät auf die Errichtung einer Lehrkanzel für das Militärrecht gedrungen hatten, zumal diese Materie nicht nur während des Krieges ,ausserordentliche Bedeutung gewonnen" habe, sondern diese Bedeutung wohl auch in der Nachkriegszeit behalten werde. Kelsen habe "sowohl in lehramtlicher als wissenschaftlicher Beziehung vorzügliche Erfolge aufzuweisen und besitze auch die „Eignung für die von der Heeresverwaltung gewünschte Behandlung des Heeresrechtes im vollen Masse, weshalb er für diese Stelle geradezu prädestiniert sei. $\mathrm{Zu}$ gleich thematisierte der Minister die offene Lehrkanzel in Czernowitz, für die Kelsen ja auch an erster Stelle genannt war und schlug vor, hier den Zweitgereihten, Pitamic, zu ernennen - was auch erfolgte. Beide sollten ihre Ämter mit 1. Oktober 1918 antreten - Kelsen in Wien, Pitamic in Czernowitz. ${ }^{43}$ Letzteres kam dann infolge des Zusammenbruchs der Monarchie nicht zustande.

Dieses Ende erlebte Kelsen aus nächster Nähe. Als Verbindungsmann zwischen Kriegsministerium und der sogenannten "Meinl-Gruppe“, einem Pazifistenzirkel rund um den Industriellen Julius Meinl II., hatte er Heinrich Lammasch bei dessen Bemühungen, die Monarchie zu retten, unterstützt. Als Lammasch jedoch am 27. Oktober zum - letzten - k.k. Ministerpräsi-

${ }^{43}$ ÖStA, AVA, Unterricht Allg., Univ.Wien, Karton 612, Personalakt Pisko Oskar, Z 23478/1918. 
denten ernannt wurde, lehnte Kelsen eine ihm angebotene höhere Beamtenstellung ab. ${ }^{44}$ Stattdessen war er knapp zwei Wochen später bereits für die junge Republik tätig, indem er die ersten juristischen Gutachten über die völkerrechtliche Situation des neuen Staates verfasste. Insgesamt drei Jahre, von November 1918 bis Dezember 1921, war Kelsen neben seiner Anstellung an der Universität als wissenschaftlicher Mitarbeiter in der Staatskanzlei (ab 1920: Bundeskanzleramt) tätig und wurde so zum Architekten der österreichischen Bundesverfassung. ${ }^{45}$

\section{Vom außerordentlichen zum ordentlichen Professor}

Kelsen war also ein "aufgehender Stern“, als Edmund Bernatzik am 30. März 1919 unerwartet an einem Herzschlag starb. Kelsen hatte gute Chancen, Nachfolger Bernatziks zu werden und so vom außerordentlichen zum ordentlichen Professor aufzusteigen. Allerdings gab es durchaus ernst zu nehmende Konkurrenten, wie insbesondere den schon genannten Rudolf von Laun, Extraordinarius für Verwaltungsrecht in Wien, der nach Ausrufung der Republik seine aristokratischen Wurzeln schnell abgestreift hatte, der sozialdemokratischen Partei beigetreten und zum Mitglied der österreichischen Delegation bei den Friedensverhandlungen in St. Germain bestellt worden war. ${ }^{46}$

Vielleicht aber hatte es Laun in seinen Bemühungen, sich an die neuen Verhältnisse anzupassen, ein wenig übertrieben. Anfang 1919 hatte die von Sowjetrussland ausgehende Rätebewegung auch Österreich erfasst, ${ }^{47}$ und in einer Professorenversammlung Mitte April sprach sich Laun dafür aus, dass sich auch die Univer-

\footnotetext{
${ }^{44}$ KELSEN, Autobiographie 51-54.

${ }^{45}$ Dazu OlechOWSKI, Beitrag Kelsens.

${ }^{46} \mathrm{Vgl}$. zu ihm BISKUP, Laun, besonders 76ff.

${ }^{47}$ HautManN, Rätebewegung.
}

sität an Wahlen zu einem Arbeiterrat beteiligen solle, während Kelsen erklärte, „dass die Verfassung der Arbeiterraete mit dem Grundsatz der Freiheit der Wissenschaft unvereinbar sei [...] Ich glaube, dass meine Rede sehr dazu beigetragen hat, dass die Beteiligung an der Wahl in den Arbeiterrat abgelehnt wurde." ${ }^{48}$ Allerdings musste Kelsen auch befürchten, dass er nun seine Chancen bei der sozialdemokratisch geführten Unterrichtsverwaltung verspielt hatte.

Dem aber war nicht so. Tatsächlich hatten die Sozialdemokraten recht wenig Interesse an einem Rätesystem; sie vertrauten lieber den jungen demokratischen Strukturen. Dagegen hatte Kelsen mit seiner Wortmeldung bei den konservativen Professoren gegenüber Laun gewonnen. Als im Juni 1919 die Berufungskommission über die Nachfolge Bernatziks beriet, verfasste der zweite Lehrstuhlinhaber, Adolf Menzel, am 15. Juni 1919 ein Gutachten, das ganz auf Kelsen zugeschnitten war und den übrigen Mitbewerbern nur wenig Raum ließ. So wie in Graz 1916, so wurde auch nun der "Bewerbermarkt" auf Österreicher zugeschnitten; insbesondere Ludwig Spiegel aus Prag käme nicht in Frage, weil die tschechoslowakischen Gehälter viel besser seien als in Deutschösterreich, aber auch, weil sich die deutsche Universität in Prag momentan in einer schwierigen Situation befinde, in der Spiegel sie nicht im Stich lassen dürfe. ${ }^{49}$ Aber auch die österreichischen Mitbewerber wurden eher pauschal abgefertigt. Layer selbst sei „kein sehr fruchtbarer Schriftsteller", er habe seit seiner "allerdings vorzüglichen“ Monographie über das Enteignungsrecht kaum etwas Größe-

\footnotetext{
${ }^{48}$ KeLSEN, Autobiographie 58. Vgl. dazu auch den Bericht über die Professorenversammlung in der Arbeiter-Zeitung Nr. 114 vom 26.4.1919, 5, in dem aber weder Kelsen noch Laun - dafür aber u.a. Ernst von Schwind - Erwähnung finden; offenbar hat Kelsen selbst die Bedeutung seiner Wortmeldung überschätzt.

${ }^{49} \mathrm{Vgl}$. dazu OBERKOFLER, Ludwig Spiegel, besonders $81 \mathrm{ff}$.
} 
res publiziert. ${ }^{50}$ Von Dungern hieß es plötzlich, er habe auf dem Gebiete des österreichischen Staatsrechts keine literarischen Leistungen aufzuweisen, sondern sei vorwiegend Rechtshistoriker, und bei Kulisch wurde überhaupt nur gesagt, dass er "schwerlich in Betracht" komme, ohne dies näher zu begründen. Ausführlich ging Menzel dagegen auf die Leistungen Kelsens ein und nahm dabei auch auf das Gutachten Bezug, das er schon 1911 zu Kelsens Habilitationsschrift verfasst hatte. Aber auch Kelsens Arbeiten für Staatskanzler Renner wurden kommentiert: „Die intime Kenntnis dieser legislativen Arbeiten dürfte seiner Lehrtätigkeit jedenfalls sehr zustatten kommen. ${ }^{\text {"51 }}$

Insgesamt urteilte Menzel: „Die ungewöhnliche Begabung, das grosse Lehrtalent, die Vereinigung von theoretischer Vertiefung und Kenntnis der Praxis, wie sie die Person Kelsens darbieten, lassen es gerechtfertigt erscheinen, diesen Gelehrten an erster Stelle zu nennen. “52 Er selbst wäre eigentlich dafür, Kelsen überhaupt unico loco zu nennen. Wenn aber ein Dreiervorschlag gemacht werden solle, so sei Kelsen an erster Stelle, Laun an zweiter Stelle zu nennen. Für die dritte Stelle nannte er ex aequo Hans Nawiasky, der sich in Wien habilitiert hatte und derzeit in München lehrte, sowie Leo Wittmayer, der im Handelsministerium arbeitete.

Bei den Kommissionsmitgliedern und später im Kollegium fand dieser Vorschlag breite $\mathrm{Zu}$ stimmung; der Nationalökonom Othmar Spann sprach sich ebenfalls dafür aus, Kelsen als einzigen (unico loco) zu nominieren und die zweite

\footnotetext{
${ }^{50}$ Bericht betreffend die Besetzung der erledigten Lehrkanzel des Staats- und Verwaltungsrechts vom 15. 6. 1919, pag. 14, ÖStA, AVA, Unterricht allg., Univ. Wien, Kart. 608, Rechts- und Staatswissenschaften.

${ }^{51}$ Bericht (wie Anm. 50) pag. 18.

${ }^{52}$ Bericht (wie Anm. 50) pag. 18.
}

und dritte Stelle frei zu lassen..$^{53}$ Nur der Völkerrechtler Alexander Hold-Ferneck - er war von Kelsen in dessen Habilitationsschrift scharf angegriffen worden -, meinte, es wäre besser, Kelsen und Laun gemeinsam (ex aequo) an die erste Stelle zu setzen. Schließlich, am 5. Juli, beschloss die Fakultät, Kelsen an die erste, Laun an die zweite Stelle zu setzen, die dritte Stelle wurde offen gelassen. ${ }^{54}$ Die Akten wurden dem Staatsamt für Unterricht übermittelt und von diesem in Rekordgeschwindigkeit bearbeitet; schon am 19. Juli 1919 unterzeichnete Karl Seitz in seiner Funktion als Staatsoberhaupt der Republik Deutschösterreich die Ernennung Kelsens zum „ordentlichen Professor der Rechts- und Staatswissenschaften an der Universität Wien“ ${ }^{.55}$

„Daß [...] die Wiener Fakultät Hans Kelsen Rudolf Laun vorreihte, muß als Schlüsselentscheidung für die weitere Entwicklung der österreichischen Rechtstheorie betrachtet werden, wäre doch ohne die Ernennung Kelsens die Wiener Rechtstheoretische Schule nicht in jene bestimmende Stellung gelangt. Verstärkt wurde diese Weichenstellung dadurch daß Laun Wien verließ ${ }^{\prime \prime}{ }^{6}$ Er folgte einem Ruf der neu gegründeten Universität Hamburg, während zum neuen Extraordinarius in Wien 1921 ausgerechnet Kelsens treuester Gefolgsmann, Adolf J. Merkl ernannt wurde.

Staatskanzler Renner befand sich zu jener Zeit bereits in St. Germain, um dort die Friedensver-

${ }^{53}$ Protokoll der Kommissionssitzung vom 18. 6. 1919, ÖStA, AVA, Unterricht allg., Univ. Wien, Kart. 608, Rechts- und Staatswissenschaften.

${ }^{54}$ Bericht des Dekans Carl Grünberg an das deutschösterreichische Staatsamt für Unterricht vom 7. 7. 1919, ÖStA, AVA, Unterricht allg., Univ. Wien, Kart. 608, Rechts- und Staatswissenschaften.

${ }^{55}$ Entschließung des Präsidenten der Nationalversammlung vom 19.7.1919, ÖStA, AVA, Unterricht allg., Univ. Wien, Kart. 611, Personalakt Kelsen Hans. ${ }^{56}$ GOLLER, Rechtsphilosophie 186. Dass Merkl zum förmlichen Nachfolger Launs ernannt wurde, wie von GOLLER behauptet, ist den Akten allerdings nicht zu entnehmen. 
handlungen mit den Siegermächten des Ersten Weltkrieges zu führen. Dort erhielt er Post von seiner Sekretärin Anna Pölzer, die ihn von der Ernennung Kelsens informierte: „[...] Eine Neuigkeit! Prof. Kelsen hat die Kanzel vom Bernatzik bekommen. Viele ärgern sich darüber, da die Besetzung der Kanzeln oft Monate gedauert haben [sic!]. Die Besetzung der Bernatzikschen Kanzel durch Kelsen war in acht Tagen perfekt. Laun ist unglücklich darüber. Wie mir Polizeirat Pollak (Kommunistenreferat) mitteilt, soll Laun deshalb die Kanzel nicht bekommen haben, weil er dem Professorenkollegium zu radikal erschien, das heißt: Grünberg hat ihn seinerzeit als Kandidat für den Arbeiterrat vorgeschlagen. Man sieht: Ein Arier kommt nicht auf. ${ }^{\text {}}{ }^{57}$

Dieser letzte Satz sollte nachdenklich stimmen. Wenn selbst eine enge Vertraute Karl Renners derartiges sagen konnte - was war dann allgemein die Meinung über Kelsen? Es ist dieser Brief nur ein erstes Indiz dafür, dass seine Professur von Anfang an nicht unumstritten war; und tatsächlich blieben insbesondere die Professoren Schwind und Hold-Ferneck - nicht nur aus fachlich-wissenschaftlichen, sondern auch aus antisemitischen Beweggründen - weiterhin erbitterte Gegner Kelsens, die später ganze Monographien gegen ihn schrieben und auch immer wieder versuchten, die Habilitationen von Kelsen-Schülern zu verhindern. ${ }^{58}$ Es waren diese Fakultätskämpfe mitursächlich dafür, dass Kelsen schließlich, im Jahre 1930, die Universität Wien verließ und einen Ruf nach Köln annahm. An seiner Heimatuniversität blieb seine Lehre umstritten - bis zum heutigen Tag. ${ }^{59}$

\footnotetext{
${ }^{57}$ Zitiert nach ScHMITZ, Karl Renners Briefe 32; Hervorhebungen im Original. Die Ausstellerin des Briefes wird dort irrtümlich als Amalia Pölzer bezeichnet.

${ }^{58}$ KORB, Kelsens Kritiker 103f.; OLECHOWSKI, EHS, STAUDIGL-CIECHOWICZ, Fakultät 491-493.

${ }^{59}$ Siehe dazu künftig OLECHOWSKI, Jurisprudenz oder Rechtswissenschaft?
}

\section{Korrespondenz:}

Prof. Dr. Thomas Olechowski

Universität Wien, Institut für Rechts- und

Verfassungsgeschichte, Leiter der Abt. KRGÖ

Strohgasse $45 / 2 d$

1030 Wien

thomas.olechowski@univie.ac.at

\section{Literatur:}

ANONYMUS, Hauke Franz, in: ÖBL, Bd. 2 (Wien 1959) 213

Rainer BISKUP, Staatsrechtslehrer zwischen Republik und Diktatur: Rudolf Laun (1882-1975) (Hamburg 2010).

Jürgen BusCH, Hans Kelsen im Ersten Weltkrieg. Achsenzeit einer Weltkarriere, in: Robert WALTER, Werner OGRIS, Thomas OlECHOWSKI (Hgg.), Hans Kelsen: Leben - Werk - Wirksamkeit (= Schriftenreihe des Hans Kelsen-Instituts 32, Wien 2009) 5780.

Eugen EHRLICH, Grundlegung der Soziologie des Rechts (1913, ND München-Leipzig 1929).

Eugen EHRLICH, Entgegnung (1916), Wiederabdruck in: Klaus LÜDERSSEN (Hg.), Rechtssoziologie und Rechtswissenschaft (Baden-Baden 2003) 57-65.

Eugen EHRLICH, Replik (1916/17), Wiederabdruck in: Klaus LÜDERSSEN (Hg.), Rechtssoziologie und Rechtswissenschaft (Baden-Baden 2003) 75-76.

Tamara EHS, Hans Kelsen und politische Bildung im modernen Staat (= Schriftenreihe des Hans KelsenInstituts 29, Wien 2007).

Peter GOLLER, Naturrecht, Rechtsphilosophie oder Rechtstheorie? Zur Geschichte der Rechtsphilosophie an Österreichs Universitäten (1848-1945) (= Rechts- und Sozialwissenschaftliche Reihe 18, Frankfurt am Main u.a. 1997).

Notker HAMmersteIN, Die Johann Wolfgang GoetheUniversität Frankfurt am Main, Bd. 1: 1914-1950 (Neuwied-Frankfurt am Main 1989).

Hans HAUTMANN, Die österreichische Rätebewegung 1918/19, in: politicum 102 (2007) 37-40.

Wladimir HeKaJLlo, Kulisch Max, in: ÖBL, Bd. 4 (Wien 1969) 341.

Matthias JeSTAEDT, Editorischer Bericht „Hauptprobleme der Staatsrechtslehre“, in: Hans Kelsen Werke, hg. v. Matthias JestAedt, Bd. 2 (Tübingen 2008) 881-913.

Hans KELSEN, Hauptprobleme der Staatsrechtslehre entwickelt aus der Lehre vom Rechtssatze (1911), 
in: Hans Kelsen Werke, hg. v. Matthias JestAedt, Bd. 2 (Tübingen 2008) 21-878.

Hans KeLSEN, Über Grenzen zwischen juristischer und soziologischer Methode (1911), in: Hans Kelsen Werke, hg. v. Matthias JestaEdT, Bd. 3 (Tübingen 2010) 22-55.

Hans KeLSEN, Eine Grundlegung der Rechtssoziologie (1914/1915), in: Hans Kelsen Werke, hg. v. Matthias JESTAEDT, Bd. 3 (Tübingen 2010) 317-358.

Hans Kelsen, Replik (1916), in: Hans Kelsen Werke, hg. v. Matthias JeSTAEDT, Bd. 3 (Tübingen 2010) 607-612.

Hans KELSEN, Schlusswort (1916/1917), in: Hans Kelsen Werke, hg. v. Matthias JestAEdT, Bd. 3 (Tübingen 2010) 613-614.

Hans KELSEN, Autobiographie (1947), in: Hans Kelsen Werke, hg. v. Matthias JeSTAEDT, Bd. 1 (Tübingen 2007) 29-91.

Axel-Johannes KORB, Kelsens Kritiker. Ein Beitrag zur Geschichte der Rechts- und Staatstheorie (19111934) (= Grundlagen der Rechtswissenschaft 13, Tübingen 2010).

Gerhard OвERKOFLER, Ludwig Spiegel und Kleo Pleyer. Deutsche Misere in der Biografie zweier sudetendeutscher Intellektueller (Innsbruck-WienBozen 2012).

Gerhard OBERKOFLER, Eduard RABOFSKY, Hans Kelsen im Kriegseinsatz der k.u.k. Wehrmacht (= Rechtshistorische Reihe 58, Frankfurt am Main u.a. 1988).

Thomas OLECHOWSKI, Rechtsphilosophie gegen Rechtsgeschichte? Ein Juristenstreit aus der Zwischenkriegszeit an der Wiener Rechtsfakultät, in: Gerald KOHL, Christian NeschwARA, Thomas SIMON (Hgg.), Festschrift für Wilhelm Brauneder zum 65. Geburtstag. Rechtsgeschichte mit internationaler Perspektive (Wien 2008) 425-442.
Thomas OlechOWSKI, Der Beitrag Hans Kelsens zur österreichischen Bundesverfassung, in: Robert WALTER, Werner OGRIS, Thomas OLECHOWSKI (Hgg.), Hans Kelsen. Leben - Werk - Wirksamkeit (= Schriftenreihe des Hans Kelsen-Instituts 32, Wien 2009) 211-230.

Thomas OlECHOWSKI, Jurisprudenz oder Rechtswissenschaft? Zur Entwicklung des wissenschaftlichen Leitbilds der juristischen Fakultät der Universität Wien seit 1852, in: Karl A. FRÖsCHL u.a. (Hgg.), Reflexive Innensichten aus der Universität (=650 Jahre Universität Wien 4, Wien 2015, im Druck).

Thomas OlechOWSKI, Tamara EHS, Kamila STAUDIGLCIECHOWICZ, Die Wiener Rechts- und Staatswissenschaftliche Fakultät 1918-1938 (= Schriften des Archivs der Universität Wien 19, Göttingen 2014).

Marijan PAVČNIK, Leonid Pitamic, in: Robert WALTER, Clemens JAbloner, Klaus ZelenY (Hgg.), Der Kreis um Hans Kelsen (= Schriftenreihe es Hans Kelsen-Instituts 30, Wien 2008) 324-350.

Georg ScHMITZ, Karl Renners Briefe aus Saint Germain und ihre rechtspolitischen Folgen (=Schriftenreihe des Hans Kelsen-Instituts 16, Wien 1991).

Michael STOLLEIS, Geschichte des öffentlichen Rechts in Deutschland, Bd. II: Staatsrechtslehre und Verwaltungswissenschaft 1800-1914 (München 1992).

Robert WALTER, Die Lehre des öffentlichen Rechts an der Karl-Franzens-Universität zu Graz von 1827 bis 1938, in: JB1 88 (1966) 546-553.

Robert WALTER, Die Lehre des Verfassungs- und Verwaltungsrechts an der Universität Wien von 1810-1938, in: JB1 110 (1988) 609-624. 